\title{
IDO/TDO Inhibitor HTI-1090
}

National Cancer Institute

\section{Source}

National Cancer Institute. IDO/TDO Inhibitor HTI-1090. NCI Thesaurus. Code C151945.

An orally available inhibitor of indoleamine 2,3-dioxygenase 1 (IDO1; IDO-1) and the kynurenine-producing hepatic enzyme tryptophan 2,3-dioxygenase (TDO), with potential immunomodulating and antineoplastic activities. Upon administration, IDO1/TDO inhibitor HTI-1090 specifically targets and binds to both IDO1, a cytosolic enzyme responsible for the oxidation of the amino acid tryptophan into the immunosuppressive metabolite kynurenine, and TDO, a hepatic enzyme catalyzing the first step of tryptophan degradation. By inhibiting IDO1 and TDO, HTI-1090 decreases kynurenine levels in tumor cells, restores tryptophan and promotes the proliferation and activation of various immune cells, including dendritic cells (DCs), natural killer (NK) cells and Tlymphocytes. This reduces the number of tumor-associated regulatory T-cells (T regs) and activates the immune system to induce a cytotoxic T-lymphocyte $(C T L)$ response against the IDO1/TDO-expressing tumor cells, thereby inhibiting the growth of the tumor cells. IDO1 and TDO, both overexpressed by multiple tumor cell types, play important roles in immunosuppression and the promotion of tumor cell survival and proliferation. Tryptophan depletion inhibits T-lymphocyte proliferation and activation, and subsequently suppresses the immune system. 